\title{
Identification of continuous-time systems from samples of input-output data: An introduction
}

\author{
NARESH K SINHA \\ Department of Electrical and Computer Engineering, McMaster University, \\ Hamilton, Ontario, Canada L8S 4L7 \\ e-mail: sinha@ece.eng.mcmaster.ca; naresh.sinha@ieee.org
}

\begin{abstract}
This paper presents an introductory survey of the methods that have been developed for identification of continuous-time systems from samples of input-output data. The two basic approaches may be described as (i) the indirect method, where first a discrete-time model is estimated from the sampled data and then an equivalent continuous-time model is calculated, and (ii) the direct method based on concepts of approximate numerical integration, where a continuous-time model is obtained directly without going through the intermediate step of first determining a discrete-time model.
\end{abstract}

Keywords. Continuous-time systems; approximate numerical integration; discrete-time model.

\section{Introduction}

Identification of process parameters for control purposes must often be done using a digital computer, from samples of input-output observations. On the other hand, the process is usually of continuous-time nature, and its dynamical model is most aptly described in terms of differential equations. Thus our problem may be stated as determining a continuous-time model from samples of input-output data.

During the past few decades, several approaches have been developed (Sinha 1972; Hsia 1972; Sinha \& Kuszta 1983; Sinha \& Rao 1991). For the sake of simplicity, these can be classified as (i) direct methods, and (ii) indirect methods. Methods belonging to the first type attempt to estimate the parameters of a continuous-time model directly from the samples of the observations, mostly using some type of numerical integration. In methods of the latter group, the problem is conveniently divided into two subproblems. The first subproblem consists of estimating the parameters of a discrete-time model from the samples of the input-output observations while the second subproblem consists of determining a suitable continuous-time model that is equivalent to the discrete-time model obtained for a given sampling interval.

The object of this paper is to give an overall view of both types of methods. The relative advantages and disadvantages of the various methods will be discussed so that a reader gets an up-to-date picture of the state of affairs. We shall start by first presenting the background 
information. This will be followed by a description of the indirect methods. Then we shall go on to direct methods, and finally we shall present an overall view.

\section{Background}

To maintain generality, it will be assumed that the continuous-time system to be identified is of the multivariable type, and can be represented by linear state-space equations of the following form.

$$
\begin{aligned}
\dot{\boldsymbol{x}}(t) & =\boldsymbol{A} \boldsymbol{x}(t)+\boldsymbol{B} \boldsymbol{u}(t), \\
\boldsymbol{y}(t) & =\boldsymbol{C} \boldsymbol{x}(t)+\boldsymbol{D} \boldsymbol{u}(t),
\end{aligned}
$$

where $\boldsymbol{x}$ is the $n$-dimensional state vector, $\boldsymbol{u}$ is the $m$-dimensional input vector and $\boldsymbol{y}$ is the $p$-dimensional output vector. In the general case, both $m$ and $p$ are smaller than $n$. The special case of single-input single-output system occurs when $m=p=1$.

Assuming that the state is known at $t=t_{1}$, its value at $t_{2}>t_{1}$ is readily obtained as

$$
\boldsymbol{x}\left(t_{2}\right)=e^{A\left(t_{2}-t_{1}\right)} \boldsymbol{x}\left(t_{1}\right)+\int_{t_{1}}^{t_{2}} e^{A\left(t_{2}-\tau\right)} \boldsymbol{B} \boldsymbol{u}(\tau) \mathrm{d} \tau
$$

If the sampling interval is $T$, and the values of $t_{1}$ and $t_{2}$ are selected as shown below

$$
\begin{aligned}
& t_{1}=k T, \\
& t_{2}=(k+1) T,
\end{aligned}
$$

where $k$ is any integer, then (2) can be written as

$$
\boldsymbol{x}_{k+1}=\boldsymbol{F} \boldsymbol{x}_{k}+\boldsymbol{G} \boldsymbol{u}_{k},
$$

where

$$
\begin{aligned}
& \boldsymbol{x}_{i} \triangleq \boldsymbol{x}(i T), \\
& \boldsymbol{u}_{i} \triangleq \boldsymbol{u}(i T), \\
& \boldsymbol{F}=e^{A T}, \\
& \boldsymbol{G}=\int_{0}^{T} e^{A \tau} \mathrm{d} \tau \boldsymbol{B} .
\end{aligned}
$$

Note that in the derivation of (5) it has been assumed that the input $u(t)$ is held constant between the sampling instants, as will be the case if a zero-order hold is utilized with the sampler. If this is not so, then the expressions for $\boldsymbol{F}$ and $\boldsymbol{G}$ will need modification, based on the knowledge of how the input varies during the sampling interval. Since this knowledge is seldom available in most practical cases, one may still use (5) as an approximation. This approximation will be quite good if the sampling interval is fairly small. Furthermore, the matrices $\boldsymbol{F}$ and $\boldsymbol{G}$ can be easily calculated from the following power series expansions (Sinha \& Lastman 1991).

$$
\boldsymbol{F}=\sum_{i=0}^{\infty} \frac{(\boldsymbol{A} T)^{i}}{i !}
$$


and

$$
\boldsymbol{G}=\left[\sum_{i=0}^{\infty} \frac{(\boldsymbol{A} T)^{i}}{(i+1) !}\right] \boldsymbol{B} T .
$$

In practice, the series can be truncated after a certain number of terms to obtain the desired accuracy. It can be shown that if the sampling interval $T$ is selected so that the spectral radius of $\boldsymbol{A}$ is less than $1 /(2 T)$ then one can truncate the series after 12 terms with an error of less than $10^{-12}$ in the elements of $\boldsymbol{F}$ and $\boldsymbol{G}$. Since these equations are exact if the input is a step function, it is customary to call this the step-invariant transformation.

Further improvement in the approximation can be obtained by assuming that the input varies linearly between the sampling instants. This is called the ramp-invariant transformation, since it is exact for a ramp input. The equivalent discrete-time model is similar to that obtained for the step-invariant case, but now (4) is replaced by the following (Bingulac \& Sinha 1989)

$$
\boldsymbol{x}_{k+1}=\boldsymbol{F} \boldsymbol{x}_{k}+\boldsymbol{G}_{0} \boldsymbol{u}_{k}+\boldsymbol{G}_{1} \boldsymbol{u}_{k+1},
$$

where $\boldsymbol{F}$ is as given in (6) while $\boldsymbol{G}_{0}$ and $\boldsymbol{G}_{1}$ are calculated using the following series.

$$
\boldsymbol{G}_{01}=\left[\sum_{i=0}^{\infty}(i+1) \frac{(\boldsymbol{A} T)^{i}}{(i+2) !}\right] \boldsymbol{B} T,
$$

and

$$
\boldsymbol{G}_{1}=\left[\sum_{i=0}^{\infty} \frac{(\boldsymbol{A} T)^{i}}{(i+2) !}\right] \boldsymbol{B} T
$$

\section{Statement of the problem}

The problem of system identification may now be stated as the estimation of the elements of the matrices $\boldsymbol{A}, \boldsymbol{B}, \boldsymbol{C}$ and $\boldsymbol{D}$ from a record of the samples of the input output data $\boldsymbol{u}(k T)$ and $\boldsymbol{y}(k T)$, for $k=0,1,2, \ldots, N$, where $N$ is a suitable large number. It may be noted that the matrix $\boldsymbol{D}$ represents direct coupling between the input and the output, and will be zero for strictly proper transfer functions. Without any loss of generality, this will be assumed to be the case.

It should be noted that none of the matrices $\boldsymbol{A}, \boldsymbol{B}$, and $\boldsymbol{C}$ in (1) or the matrix $\boldsymbol{F}$ in (6) are unique for a system with a given input-output description. Assuming a special canonical form for the system state equations in either the continuous-time or the equivalent discretetime models overcomes this problem and also minimizes the number of parameters to be estimated. It should also be noted that it is tacitly assumed that the order of the linear statespace model is known, and that the sampling interval has been suitably selected. In practice, both of these are important, and have been subjects of considerable research (ElSherief \& Sinha 1979; Puthenpura \& Sinha 1985; Sinha \& Puthenpura 1985).

The problem is further complicated by the fact that the available data are usually contaminated with random noise that are produced either by disturbances or introduced in data acquisition and measurement. The literature on system identification abounds in papers devoted to methods for estimating the parameters in the presence of noise (see Sinha \& Kuszta 1983 for a detailed list of references). 


\section{The indirect approach}

As stated earlier, it is convenient to divide the problem into two subproblems. The first of these is the determination of a suitable discrete-time model from the samples of the input-output data; due to the inevitable presence of noise in the measured data, this problem is stochastic in nature. The second is the determination of an equivalent continuous-time model. The main advantage of this approach is that considerable literature is available on the first part of the problem. Furthermore, the second subproblem is essentially deterministic in nature. The first part of the problem has been discussed thoroughly in the literature but the second part has received less attention. Hence, we shall present a very short review of the first part, and then describe the second part in greater detail.

\subsection{Estimation of a discrete-time model from the samples of input-output data}

There are several approaches to estimating a discrete-time model from the samples of input-output data (Guidorzi 1975; Tse \& Weinert 1975; Kung 1978; Sinha et al 1978; Sinha \& Kwong 1979; El-Sherief \& Sinha 1979, 1982). Four of these methods have been described in detail in references (El-Sherief \& Sinha 1979a, c), which state that four different models can be used. Although these models are equivalent and one can easily transform any of them to another form, each of these has certain special features and advantages relative to the others. These models are:

(a) the canonical state-space representation,

(b) the input-output difference equation model (the matrix fraction representation),

(c) the impulse response matrix representation, and

(d) the transfer function matrix representation.

The input-output difference equation model or the equivalent matrix-fraction model has the smallest number of parameters to be estimated, as in the case of canonical state-space models. It has been shown (Sinha \& Kuszta 1983) that, in each case, the problem of least squares estimation of the model parameters reduces to solving a set of linear simultaneous equations which can be written in the following form,

$$
\boldsymbol{H} \theta=\boldsymbol{Y},
$$

where $\boldsymbol{H}$ is a matrix consisting of the past values of the input and the output vectors, arranged in a particular order, $\boldsymbol{\theta}$ is the vector of unknown parameters and $\boldsymbol{Y}$ is a vector obtained by concatenation of the vectors in the output sequence. The matrix $\boldsymbol{H}$ is rectangular with many more rows than columns so that (11) is over-determined. Consequently, one can obtain a least-squares estimate of the parameter vector from (11) by using either the matrix pseudo-inverse (Sinha \& Kuszta 1983) or the singular value decomposition (Kung 1978).

It is well-known that although the least-squares solution obtained from (11) is reasonably good when the noise level is low, the estimates tend to be biased and inconsistent for higher levels of noise when the state-space or matrix-fraction model is used. Use of the impulseresponse matrix eliminates the bias, but at the expense of considerably increasing the dimension of the parameter vector (Sinha et al 1978). The reason is that theoretically the impulse-response sequence is infinite-dimensional, although it is possible to use finite numbers of terms in the case of stable systems since the terms slowly approach zero as one 
moves away from the initial time. The least-squares solution can be consistent and unbiased, if one uses estimates of auto-correlation of the input sequence and cross-correlation between the input and output sequences in (11) instead of the input and output sequences (Sinha \& Kuszta 1983). Alternatively, one may include parameters of a noise model with the system model in (11).

We shall leave out the details here and only state that, using one of these methods, it is possible to obtain a suitable discrete-time model from the input-output data. For details the interested reader can look into the references cited above. Now we can go into the second sub-problem, that of obtaining an equivalent continuous-time model obtained by using any of the methods mentioned above.

\subsection{Transformation of discrete-time models into equivalent continuous-time models}

As stated earlier, this is a deterministic problem. It is assumed that the sampling interval is selected suitably, otherwise there is no unique solution to the problem. In order that there be no loss of information in the sampling process, the sampling theorem requires that the sampling frequency be at least twice the largest frequency component of the signal being sampled. Since most physical systems to be identified are stable and of low-pass nature, the above requirement is equivalent to making the sampling frequency at least twice the largest natural frequency of the system. In other words, the sampling interval, $T$, should be selected such that the spectral radius of the matrix $\boldsymbol{A}$ should be less than $1 / T$.

It may be pointed out that in practice it is seldom a good idea to make the sampling interval smaller than one-fifth of the maximum value obtained from the relationship given above, since this may make the matrix $\boldsymbol{H}^{T} \boldsymbol{H}$ ill-conditioned and thus cause numerical problems in the evaluation of the least-squares solution to (11). Here we shall assume that this condition has been met and the estimated discrete-time model is satisfactory.

Thus, the second sub-problem is essentially the determination of the system matrices $\boldsymbol{A}$, and $\boldsymbol{B}$ from the knowledge of $\boldsymbol{F}$ and $\boldsymbol{G}$. It follows from (5) that this essentially requires the calculation of the natural logarithm of a square matrix.

Several methods have been proposed by different authors for evaluation of the natural logarithm of a square matrix (Strmčnik \& Bremšak 1979; Sinha \& Lastman 1981, 1982, 1991; Raol et al 1987; Lastman \& Sinha 1991). We shall describe here an efficient algorithm for determining $\boldsymbol{A}$ and $\boldsymbol{B}$ for given $\boldsymbol{F}, \boldsymbol{G}$ and $T$, which does not require matrix inversion (Raol et al 1987), and is numerically robust.

(i) Determine $\boldsymbol{L}=\boldsymbol{F}-\boldsymbol{I}$.

(ii) Set $\boldsymbol{P}^{(1)}=\boldsymbol{I}, \boldsymbol{M}^{(1)}=\boldsymbol{I}$, and $i=1$,

(iii) Perform the following computations recursively,

$$
\begin{gathered}
\boldsymbol{M}^{(i+1)}=\frac{-i \boldsymbol{L} \boldsymbol{M}^{(i)}}{i+1}, \\
\boldsymbol{P}^{(i+1)}=\boldsymbol{P}^{(i)}+M^{(i+1)} . \\
\text { replace } i \text { with } i+1,
\end{gathered}
$$

until $i=m$ (some predetermined value) or the change in $\boldsymbol{P}^{(i)}$ is negligible.

(iv) Finally, $\boldsymbol{A} T=\boldsymbol{P L}$ and $\boldsymbol{B} T=\boldsymbol{P G}$. 


\section{The direct approach}

In this case the samples of input-output data are used to directly obtain an estimate of the continuous-time model. The idea behind all the methods used for this purpose can be best understood by obtaining the integral of the state equation (1) with respect to time during the interval $t=i T$ to $t=(i+1) T$, where $i$ is any integer, and $T$ is the sampling interval. This leads to the following equation

$$
\boldsymbol{x}(i+1)-\boldsymbol{x}(i)=\int_{t=i T}^{(i+1) T} \boldsymbol{A} x(t) \mathrm{d} t+\int_{t=i T}^{(i+1) T} \boldsymbol{B} u(t) \mathrm{d} t
$$

Since $\boldsymbol{A}$ and $\boldsymbol{B}$ are constant matrices, they can be taken outside the integral sign. Thus, if the integrals of the system state and the input between the sampling instants are available, we can concatenate the data for $i=0$ to $N$, where $N$ is some large number, to obtain a set of linear simultaneous equations where the unknowns are the elements of the matrices $\boldsymbol{A}$ and $\boldsymbol{B}$. It is evident that the number of unknowns can be significantly reduced if the state equations are in a suitable canonical form. For example, use of the observer canonical form significantly reduces the number of unknown elements in $\boldsymbol{A}$, whereas the elements of $\boldsymbol{C}$ are already known.

The main problem with this method is the requirement that all the states of the system be available for measurement. Since this is seldom the case in practice, we need some modification. This difficulty can be easily removed if the state equations are written in diagonal form (or Jordan form if some eigenvalues are repeated). Thus, for a single-input single-output system, we have

$$
\begin{aligned}
\boldsymbol{A} & =\operatorname{diag}\left[\lambda_{1}, \lambda_{2}, \ldots, \lambda_{n}\right] \\
\boldsymbol{B}^{T} & =\left[\bar{b}_{1} \bar{b}_{2} \cdots \bar{b}_{n}\right] \\
\boldsymbol{C}^{T} & =\left[\begin{array}{lll}
1 & 1 & \cdots
\end{array}\right] .
\end{aligned}
$$

The diagonal elements of the diagonal matrix $\boldsymbol{A}$ are the distinct real eigenvalues of the system. In the case of complex conjugate eigenvalues, (16) is slightly modified. For example, if there is a pair of eigenvalues $\alpha \pm j \beta$, then $\boldsymbol{A}$ has a diagonal block of the form

$$
\boldsymbol{A}=\left[\begin{array}{cc}
\alpha & \beta \\
-\beta & \alpha
\end{array}\right]
$$

We shall now write (15) as

$$
x(i+1)-x(i)=\boldsymbol{A} I_{x}(i)+B I_{u}(i),
$$

where

$$
\boldsymbol{I}_{\boldsymbol{x}}(i)=\int_{t=i T}^{(i+1)^{T}} \boldsymbol{x}(t) \mathrm{d} t
$$

and

$$
\boldsymbol{I}_{\boldsymbol{u}}(i)=\int_{t=i T}^{(i+1) T} \boldsymbol{u}(t) \mathrm{d} t
$$


In practice, however, we have only the samples of the input-output data. Thus we must resort to approximating the integrals indicated in (21) and (22) in terms of the samples available. Several methods for this purpose have been discussed by Dai \& Sinha (1991). These include the mid-point rule, the trapezoidal rule, Simpson's rule and other NewtonCoates formulas.

For example, if we use the simple trapezoidal rule of integration, we obtain

$$
\boldsymbol{I}_{\boldsymbol{x}}(i)=\frac{T}{2}[\boldsymbol{x}(i+1)+\boldsymbol{x}(i)],
$$

and

$$
\boldsymbol{I}_{\boldsymbol{u}}(i)=\frac{T}{2}[\boldsymbol{u}(i+1)+\boldsymbol{u}(i)]
$$

Substituting these approximations for the integrals we can rearrange (20) as

$$
\left(\boldsymbol{I}-\frac{\boldsymbol{A} T}{2}\right) \boldsymbol{x}(i+1)=\left(\boldsymbol{I}+\frac{\boldsymbol{A} T}{2}\right) \boldsymbol{x}(i)+\frac{\boldsymbol{B} T}{2}[u(i+1)+u(i)] .
$$

We can now exploit the diagonal form of the matrix $\boldsymbol{A}$ to rearrange (25) as

$$
x(i+1)=\operatorname{diag}\left[f_{1} f_{2} \cdots f_{n}\right] x(i)+G^{T}[u(i+1)+u(i)],
$$

where

$$
\begin{aligned}
f & =\left(1-\frac{\lambda_{i} T}{2}\right)^{-1}\left(1+\frac{\lambda_{i} T}{2}\right), \\
\boldsymbol{G}^{T} & =\left[\begin{array}{lll}
g_{1} & g_{2} & \cdots \\
g_{n}
\end{array}\right], \\
g_{i} & =\frac{T}{2}\left(1-\frac{\lambda_{i} T}{2}\right)^{-1} b_{i} .
\end{aligned}
$$

If we now premultiply both sides of (26) with $\boldsymbol{C}$, we get the following relationship in terms of the input and output data,

$$
y(i+1)=\sum_{i=1}^{n} \boldsymbol{C}\left(1-f_{i} z^{-1}\right)^{-1} \boldsymbol{G}^{T} \boldsymbol{w}(i+1),
$$

where $z^{-1}$ is the backward shift (unit delay) operator and

$$
w(i+1)=u(i+1)+u(i) .
$$

If all the eigenvalues of the system are real and distinct, (28) is reduced to

$$
y(i+1)=\sum_{i=1}^{n} \frac{g_{i}}{1-f_{i} z^{-1}} w(i+1) .
$$

Thus, if we define an "auxiliary" linear discrete-time system with input $w(i)$ and output $y(i)$, we can obtain the following transfer function

$$
H(z)=\sum_{i=1}^{n} \frac{g_{i}}{1-f_{i} z^{-1}}=\frac{b_{0}+b_{1} z^{-1}+\cdots+b_{n-1} z^{-n+1}}{1+a_{1} z^{-1}+\cdots+a_{n} z^{-n}} .
$$


With the help of (31), the problem of identification of continuous-time systems is reduced to the standard problem of identification of discrete-time systems, as formulated through (11). Consequently, all algorithms developed for the latter can be applied to this case. This includes methods like maximum likelihood estimation of parameters as well as the method of instrumental variables (Sinha \& Kuszta 1983).

There are many other methods, e.g. use of block pulse functions, Poisson moment functions, and other orthogonal functions, which perform approximate integration in a more sophisticated manner (Prasad \& Sinha 1983; Jiang 1987; Bohn 1991; Jiang \& Schaufelberger 1991; Wong 1991) that have been proposed for direct identification of continuoustime systems from samples of input-output data. These are described in detail in Sinha \& Rao (1991), Palanisamy \& Bhattacharya (1981) and Moonen et al $(1989,1991)$.

\section{Conclusions}

In this paper, two basic approaches to the identification of continuous-time systems from the samples of input-output data have been described. It has been tacitly assumed that the sampling period has been selected suitably. It is also known that making the sampling interval too small is almost as bad as making it too large. Thus, in practice, this choice assumes á priori knowledge of the largest natural frequency of the system.

Another approach that has attracted a lot of attention is the use of the delta operator (Ninness \& Goodwin 1991) instead of the operator $z$ in obtaining a discrete-time model, since larger sampling frequencies do not cause the same type of numerical difficulties as obtained with the use of $z$-transform models.

\section{References}

Bingulac S, Sinha N K 1989 On the identification of continuous-time systems from the samples of input-output data. Proc. Seventh Int. Conf. on Mathematical and Computer Modeling, Chicago, IL, pp 231-239

Bohn E V 1991 Methods using Walsh functions. In Identification of continuous-time systems (eds) N K Sinha, G P Rao (Dordrecht: Kluwer Academic) pp 139-158

Dai H, Sinha N K 1991 Use of numerical integration methods. In Identification of continuous-time systems (eds) N K Sinha, G P Rao (Dordrecht: Kluwer Academic) pp 259-290

El-Sherief H, Sinha N K 1979a On-line identification of linear discrete-time multivariable systems. Proc. Inst. Electr. Eng. 26: 1321-1325

El-Sherief H, Sinha N K 1979b Choice of models for the identification of linear multivariable discrete-time systems. Proc. Inst. Electr. Eng. 126: 1326-1330

El-Sherief H, Sinha N K 1979c Identification and modelling for linear multivariable discrete-time systems: A survey. J. Cybern. 9: 43-71

El-Sherief H, Sinha N K 1982 Determination of the structure of a canonical model for the identification of linear multivariable systems. IEEE Trans. Syst., Man Cybern. SMC-12: 668-673

Guidorzi R 1975 Canonical structures in the identification of multivariable systems. Automatica 11: $113-116$

Hsia T C 1972 On sampled-data approach to parameter identification of continuous-time linear systems. IEEE Trans. Autom. Control AC-17: 247-249

Jiang Z H 1987 Block pulse function approach for the identification of MIMO-systems and timedelay systems. Int. J. Syst. Sci. 18: 1711-1720

Jiang Z H, Schaufelberger W 1991 Recursive block pulse function method. In Identification of continuous-time systems (eds) N K Sinha, G P Rao (Dordrecht: Kluwer Academic) pp 205, 226 
Kung S Y 1978 A new identification and model reduction algorithm via singular value decomposition. Proc. 12th Asilomar Conference on Circuits, Systems and Computers, Pacific Grove, CA, pp. 705-714

Lastman G J, Sinha N K 1991 Infinite series for logarithm of a matrix, applied to identification of continuous-time multivariable systems from discrete-time models. Electron. Lett. 27: 1468-1469

Moonen M, De Moore B, Vandenberghe L, Vandewalle J 1989 On- and off-line identification of linear state space models. Int. J. Control 49: 219-232

Moonen M, De Moore B, Vandewalle J 1991 SVD-based subspace methods for multivariable continuous-time system identification. In Identification of continuous-time systems (eds) N K Sinha, G P Rao (Dordrecht: Kluwer Academic) pp 473-488

Ninness B M, Goodwin G C 1991 The relationship between discrete-time and continuous-time linear estimation. In Identification of continuous-time systems (eds) N K Sinha, G P Rao (Dordrecht: Kluwer Academic) pp 79-122

Palanisamy K R, Bhattacharya D K 1981 System identification via block pulse functions. Int. J. Syst. Sci. 12: 643-647

Prasad T, Sinha N K 1983 Modelling of continuous-time systems from sampled data using trapezoidal pulse functions. Proc. Int. Conf. on Systems, Man and Cybernetics. Bombay, India, pp. $117-126$

Puthenpura S, Sinha N K 1985 A procedure for determining the optimal sampling interval for system identification using a digital computer. Can. Electr. Eng. J. 10: 152-157

Raol J R, Puthenpura S, Sinha N K 1987 Algorithms for transformation of multivariable discretetime models to continuous-time models. Advances in modelling and simulation (AMSE Press) vol. 6, pp 52-62

Sinha N K 1972 Estimation of transfer function of continuous-time systems from samples of inputoutput data. Proc. Inst. Electr. Eng. 119: 612-614

Sinha N K, Kuszta B 1983 Modelling and identification of dynamic systems (New York: VonNostrand Reinhold)

Sinha N K, Kwong Y H 1979 Recursive identification of parameters of multivariable systems. Automatica 15: 471-475

Sinha N K, Lastman G J 1981 Transformation algorithm for identification of continuous-time multivariable systems from discrete data. Electron. Lett. 21: 779-780

Sinha N K, Lastman G J 1982 Identification of continuous-time multivariable systems from sampled data. Int. J. Control. 35: 263-267

Sinha N K, Lastman G J 1991 Transformation of discrete-time models. In Identification of continuous-time systems (eds) N K Sinha, G P Rao (Dordrecht: Kluwer Academic) pp 123-138

Sinha N K, Puthenpura S 1985 Choice of the sampling interval for the identification of continoustime systems from samples of input-output data. Proc. Inst. Electr. Eng. D132: 263-267

Sinha N K, Rao G P (eds) 1991 Identification of continuous-time systems (Dordrecht: Kluwer Academic)

Sinha N K, Mahalanabis A K, El-Sherief H 1978 A non-parametric approach to the identification of linear multivariable systems. Int. J. Syst. Sci. 9: 425-430

Strmčnik S, Bremšak F 1979 Some new transformation algorithms in the identification of continuous-time multivariable systems using discrete identification methods. Preprints of the 5th IFAC Symposium on Identification and System Parameter Estimation, Darmstadt, Germany, pp 397-405

Tse E, Weinert H 1975 Structure determination and parameter identification for multivariable stochastic linear systems. IEEE Trans. Autom. Control AC-20: 603-613

Wong S Y 1991 Use of the block-pulse operator. In Identification of continuous-time systems (eds) N K Sinha, G P Rao (Dordrecht: Kluwer Academic) pp 159-204 